\title{
GPU Accelerated Multispectral EO Imagery Optimised CCSDS-123 Lossless Compression Implementation
}

\author{
R. L. Davidson, C. P. Bridges \\ Surrey Space Centre \\ University of Surrey \\ Guildford, Surrey, GU2 7XH, UK \\ rebecca.davidson@surrey.ac.uk, c.p.bridges@surrey.ac.uk
}

\begin{abstract}
Continual advancements in Earth Observation (EO) optical imager payloads has led to a significant increase in the volume of multispectral data generated onboard EO satellites. As a result, a growing onboard data bottleneck need to be alleviated. One technique commonly used is onboard image compression. However, the performance of traditional space qualified processors, such as radiation hardened FPGAs, are not able to meet current nor future onboard data processing requirements. Therefore, a new high capability hardware architecture is required. In previous work a new GPU accelerated scalable heterogeneous hardware architecture for onboard data processing was proposed. In this paper, two new CUDA GPU implementations of the state-of-the-art lossless multidimensional image compression algorithm CCSDS-123, are discussed.

The first implementation is a generic CUDA implementation of the CCSDS-123 algorithm whilst the second is optimised specifically for multispectral EO imagery. Both implementations utilise image tiling to leverage an additional axis for algorithm parallelisation to increase processing throughput. The CUDA implementation and optimisation techniques deployed are discussed in the paper. In addition, compression ratio and throughput performance results are presented for each implementation. Further experimental studies into the relationships between algorithm user definable compression parameters, tile sizes, tile dimensions and the achieved compression ratio and throughput, were performed.
\end{abstract}

\section{TABLE OF CONTENTS}

1. INTRODUCTION 1

2. GPU ACCELERATED ONBOARD DATA

PROCESSING ARCHITECTURE .2

3. PREVIOUS CUDA CCSDS-123 GPU

IMPLEMENTATIONS......................................................... 3

4. NEW CUDA CCSDS-123 IMPLEMENTATION ........ 4

5. NEW MULTISPECTRAL OPTIMISED CUDA

CCSDS-123 IMPLEMENTATION

6. EXPERIMENTAL STUDIES

7. FUTURE WORK

8. SUMMARY ....9

APPENDICES 10

A. CCSDS-123 IMPLEMENTATION FEATURES

B. TEST PLATFORM SPECIFICATION.........................11

C. TEST IMAGES \& Default TeSting

PARAMETERS.

REFERENCES.

BIOGRAPHY

\section{INTRODUCTION}

Earth Observation (EO) satellites are experiencing continual advancements in optical imager payload technologies, leading to significant increases in the resolutions of images gathered from spaceborne platforms. Due to the absence of equivalent developments in satellite downlink technologies, the exponential increases in the generated data volume are forming a significant bottleneck onboard the platform. Image compression is a data encoding technique which can be used to reduce the amount of data required to represent the imagery information and is being increasingly explored to help effectively increase the data delivery throughput of EO satellites.

Specifically, lossless image compression algorithms are predominantly used in the field. This is due to the importance of data fidelity to many EO data applications, particularly as computer based image processing becomes increasingly prominent for information extraction. Currently most operational satellites implement standardised algorithms such as JPEG-LS or JPEG-2000 where both these algorithms typically achieve compression ratios of approximately 2.0 [1]. However, in order to help alleviate the increasing onboard data bottleneck, new state-of-the-art compression and processing algorithms will need to be deployed to achieve increased compression ratios onboard spaceborne platforms. Due to the inherent restrictions induced by the space environment; time, memory and computational resources are often heavily restricted when compared with terrestrial systems. Therefore, for space applications, algorithms that minimise computational complexity and resource requirements are preferred.

A literature review of an extensive range of lossless image compression algorithms has been conducted in previous work [3]. The survey was conducted with the aim of identifying suitable algorithms specifically for the implementation on spaceborne platforms for multispectral EO image compression. In the review, average compression ratio values were calculated and used as a quantitative comparison metric. The comparison of the lossless image compression algorithms surveyed highlighted the significant compression advantages that can often be gained from utilising multidimensional compression algorithms. Multidimensional image compression algorithms are those that exploit additional spectral redundancies between image bands, as shown in Figure 1. 


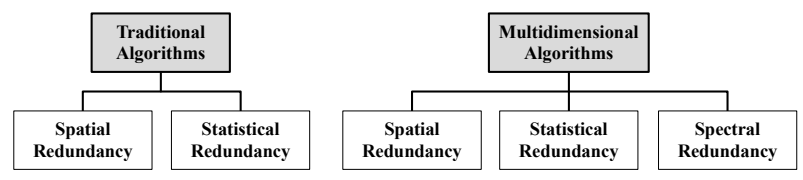

Figure 1: Lossless image compression classification [1]

Additionally, in the previous research the complexity of a number of algorithms were qualitatively assessed for their suitability for implementation in the space environment. The CCSDS-123 algorithm was identified as a suitable algorithm that provides state-of-the-art compression performance in low complexity domain. The CCSDS- 123 algorithm was standardised by the Consultative Committee for Space Data Systems (CCSDS) in 2012 [2] and is based on the Fast Lossless (FL) algorithm developed by the NASA Jet Propulsion Laboratory (JPL) in 2005 [3]. The algorithm employs a predictive based scheme incorporating information from a 3D neighbourhood of pixels to exploit both spatial and spectral redundancies. It also features a low complexity version of the least means squares algorithm and local mean subtraction to control the weightings of the prediction from each of the preceding spectral bands. As the algorithm, has been designed specifically for reduced computational complexity it is well suited for onboard implementation in a restricted hardware environment. Another characteristic of the algorithm that makes it attractive for onboard implementation is the possibility for some parallelisation for increased processing throughput. This is due to the data dependencies of the different algorithms stages, as shown in Figure 2. By exploiting the inherent parallelism of the algorithm using a suitable parallel processing hardware architecture, high data processing throughput could be achieved. In an ideal scenario, this would be equal to the data rate of the payload thus allowing the processing to be conducted in real-time.

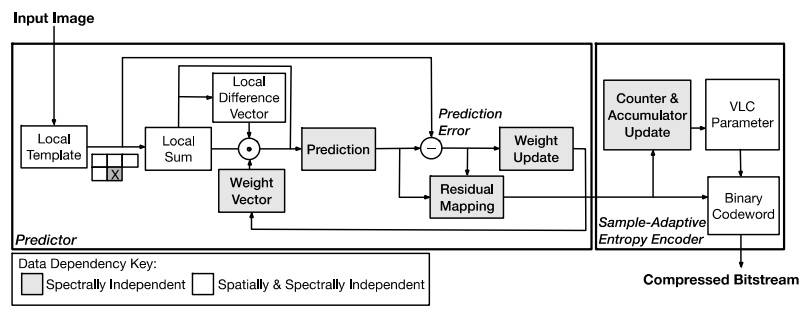

Figure 2: CCSDS-123 algorithm \& data dependencies

Traditionally in the space community radiation hardened FPGAs are used for onboard data processing. However, these processors often lag several generations behind their terrestrial counterparts in terms of computational performance. With the projected ever-increasing imager payload data volumes, computational resources are now a growing priority to help alleviate the onboard data bottleneck. Traditional space qualified processors are no longer able to meet the demands of current nor future onboard data processing system requirements [4]. Therefore, as part of previous research a new onboard data processing architecture has been proposed [1]. The new GPU accelerated scalable heterogeneous hardware architecture aims to facilitate the implementation of state-of-the-art parallel image processing and compression algorithm to help alleviate the growing onboard data bottleneck of EO satellite platforms.

The remainder of this paper is organised as follows. Section 2 provides an overview of the previously proposed GPU accelerated onboard data processing hardware architecture. Section 3 discusses the previous work on GPU implementations of the CCSDS-123 lossless image compression algorithm. Section 4 provides details on the implementation of our new CUDA CCSDS-123 software, including performance results. Section 5 discusses the optimisation of the new CUDA CCSDS-123 implementation, specifically for multispectral EO imagery. This section also includes profiling analysis, discussions on optimisation techniques and presents the final performance results. Section 6 provides the results of several new experimental studies conducted with our new CUDA implementation. It specifically addresses the relationships between input parameters such as compression modes, prediction bands and tiling dimensions and the achieved compression ratio and processing throughput of the software. Sections 7 and 8 provide an overview of the future work which is under development and an overall summary of the findings presented in this paper.

\section{GPU ACCELERATED ONBOARD DATA Processing Architecture}

Traditional space qualified processors are unable to meet the growing computational demands for onboard data processing. The relative scaling of these traditional system architectures, to try to meet the growing requirements will likely lead to unacceptable and exponential increases in power consumption, area and mass. As a result, a new heterogeneous onboard data processing architecture has been proposed in [1]. This architecture is also given in Figure 3, it has been designed to provide scalable high performance onboard data processing to address the growing data bottleneck onboard EO satellites. The architecture is discussed in detail in the previous publication [1], therefore this section will only provide a brief overview of its key features for image processing software development.

The heterogeneous nature of the architecture, which features high performance GPU devices, allows for the computationally intense image processing and compression to be offloaded from the less suitable traditional FPGA processing hardware. This facilitates the development of new high-throughput parallel processing GPU algorithms, whilst also enables the low-level, typically software intense, data manipulation and interfacing to remain on FPGA hardware, maximising the overall system performance. The backplane nature of the architecture permits both scalability of memory and processing resource but also graceful degradation with minimised single points of failure. It also allows a plug and play, IP core, based software model to be deployed, where 
new advances in image compression and processing algorithms can easily be incorporated into existing software, with minimal impact on hardware design. This also allows for greater design re-use across multiple platforms and missions. To demonstrate the advantages of the new GPU accelerated data processing architecture, a newly parallelised implementation of the CCSDS-123 image compression algorithm has been developed.

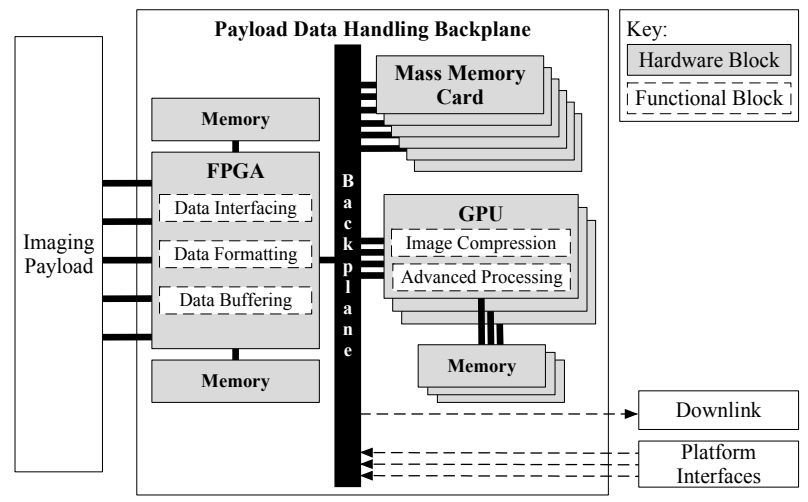

Figure 3: New onboard data processing architecture [1]

\section{Previous CUDA CCSDS-123 GPU IMPLEMENTATIONS}

The technical details of the CCSDS-123 algorithm have been covered extensively in several publications in addition to the CCSDS standard document and the original FL algorithm publication $[2,3,5,6,7]$. Therefore, the specific details of the algorithm will not be covered in this paper, but an overview of the algorithm stages and their relative data dependences can be found in Figure 2 .

Two recent publications by NASA JPL have discussed their work on the development of two GPU implementations of CCSDS-123. These works are referred to in this paper as fixed platform and mobile platform implementations respectively [6,7]. The first proposed, fixed platform, implementation deployed a "greedy style" optimisation for parallel GPU implementation, whereby the largest amount of parallelism present at each stage of the algorithm was exploited, and buffers were used between stages to store intermediate results. This approach implies that each stage of the algorithm would need to be implemented as individual kernels. The CUDA GPU memory model means that the intermediate results from each stage would need to be stored in off-chip, high latency memory. GPUs are typically composed of hundreds of computational cores that can execute thousands of threads simultaneously for fast parallel processing, making it a highly efficient architecture for the compute intensive algorithm stages. However, the implementations reliance on high latency, off-chip memory means that there is little opportunity for data re-use or efficient utilisation of low latency, on-chip memory structures [6].
The proceeding, mobile platform, implementation addressed these issues by taking an alternative parallelisation approach, whereby the parallelism exploited was restricted to that of the least parallel stage of the algorithm. As a result, the large off-chip data buffers where no longer required to store intermediate results and whilst the data flow was more akin to a serial implementation this allowed the low latency on-chip memories to be fully exploited [7]. The throughput performance achieved by these previous works are given in Table 1 for reference.

Table 1. Previously published implementation performance results - GPU used GeForce GTX 560M, Test image used Hyperspectral AVIRIS Hawaii

\begin{tabular}{|c|c|c|}
\hline Implementation & Time (ms) & Throughput (Mb/s) \\
\hline Fixed Platform [6] & 2346 & 372.14 \\
\hline $\begin{array}{c}\text { Mobile Platform } \\
\text { (1 GPU) [7] }\end{array}$ & 226 & 3862.97 \\
\hline $\begin{array}{c}\text { Mobile Platform } \\
\text { (2 GPUs) [7] }\end{array}$ & 204 & 4279.56 \\
\hline
\end{tabular}

The performance of these implementations were tested using a hyperspectral optical test image taken by the AVIRIS instrument. Currently there are two classes of multiband optical EO imagery data that are gathered from satellites, multispectral and hyperspectral. Hyperspectral data sets can be considered as an almost continuous representation of electromagnetic reflectance, as the data is gathered for a large number, in the order of several hundred, narrow spectral bands. Multispectral EO imagery on the other hand, provides electromagnetic reflectance data in a small number of discrete spectral bands, in the order of tens, but often provide significantly greater spatial resolution than hyperspectral imagers. Whilst hyperspectral data sets pose an interesting big data processing problem for the community, they are not currently widely used onboard satellites. As such the majority of EO data gathered from spaceborne platforms are currently multispectral images. The intrinsic differences between these two types of data sets can have significant impacts on both the achievable processing throughput and achievable compression ratio of an onboard image compression system. When compared with hyperspectral imagery data multispectral data has inherently reduced compressibility due to reduced correlations between spectral image bands. As the major axis for parallelisation for the CCSDS-123 algorithm is also the number of bands, it is therefore postulated that the achievable compression processing throughput will also be reduced for multispectral imagery. The focus of this research is to assess the performance of a GPU implementation of the CCSDS-123 algorithm specifically for multispectral imagery data. 


\section{NEW CUDA CCSDS-123 IMPLEMENTATION}

The operation our new CUDA CCSDS-123 implementation is shown in Figure 4, it adheres to the CCSDS-123 standard, a summary of the capabilities it provides can be found in appendix A. Figure 4 also provides information on the kernel configuration parameters and memory requirements. We have considered the lessons learned from the previous implementations [6,7] to develop this new implementation. This sections aims to provide details of new lessons learned, where the following subsections provide further details of each of the CUDA kernels developed for our CCSDS-123 implementation.

\begin{tabular}{|c|c|c|c|}
\hline Hardware & \multicolumn{2}{|c|}{ Operations } & $\begin{array}{c}\text { Memory } \\
\text { Allocation } \\
\text { (Bytes) }\end{array}$ \\
\hline Host & \multicolumn{2}{|c|}{ Load Compression Parameters } & 21 \\
\hline Host & \multicolumn{2}{|c|}{ Load Image Samples } & $\begin{array}{c}2 * \text { X Size * } \\
\text { Y Size * Z Size }\end{array}$ \\
\hline \multirow{5}{*}{ Device } & \multicolumn{2}{|c|}{$\begin{array}{l}\text { I. CCSDS-123 Kernel } \\
\text { Registers/Thread }=72\end{array}$} & \multirow{5}{*}{$\begin{array}{c}\text { Global Memory } \\
=(14 * \text { X Size } \\
\text { Y Size * Z Size }) \\
\quad+21 \\
\text { Shared Memory } \\
=8^{*} \text { Z Size } \\
\text { Weights_Length }\end{array}$} \\
\hline & Grid Size & \# Tiles & \\
\hline & Block Size & Z Size & \\
\hline & Inputs & $\begin{array}{l}\text { - Parameters } \\
\text { - Samples }\end{array}$ & \\
\hline & Outputs & \begin{tabular}{|l|} 
- Codewords \\
- Lengths \\
\end{tabular} & \\
\hline \multirow{3}{*}{ Device } & \multicolumn{2}{|c|}{ II. THRUST - Inclusive Sum } & \multirow{3}{*}{ NA } \\
\hline & Input & - Lengths & \\
\hline & Output & - Lengths & \\
\hline \multirow{5}{*}{ Device } & \multicolumn{2}{|c|}{$\begin{array}{c}\text { III. Bit Packer Kernel } \\
\text { Registers/Thread }=20\end{array}$} & \multirow{5}{*}{$\mathrm{NA}^{2}$} \\
\hline & Grid Size & 1024 & \\
\hline & Block Size & $\begin{array}{c}\text { Ceil }\{(X \text { Size * } \\
\text { Y Size* Z Size) } \\
/ 1024\}\end{array}$ & \\
\hline & Inputs & \begin{tabular}{|l|} 
- Codewords \\
- Lengths \\
\end{tabular} & \\
\hline & Outputs & $\begin{array}{l}\text { - Compressed } \\
\text { Stream } \\
\end{array}$ & \\
\hline Host & \multicolumn{2}{|c|}{ Store Compressed Stream } & Variable \\
\hline \multicolumn{4}{|c|}{$\begin{array}{l}{ }^{1} \text { Weights_Length }=\# \text { Prediction Bands }+ \text { if Prediction Mode }= \\
\text { FULL\} } 3 \text { \{ELSE\} } 0 \\
{ }^{2} \text { No new memory needs to be allocated as the memory allocated for } \\
\text { the original image samples can be reused for the Compressed } \\
\text { Stream. }\end{array}$} \\
\hline
\end{tabular}

Figure 4: CCSDS-123 GPU implementation stages

\section{CCSDS-123 Kernel}

\section{Tiling}

As shown in Figure 2 the maximum level of parallelism available at the least parallel stages of the algorithm are across the spectral dimension of the input image data. For multispectral imagery the number of bands are commonly restricted to less than 10 . Therefore, to help further increase the amount of parallelism available, and hence increase processing throughput, images can be segmented into individual image tiles. These tiles can then be independently compressed, providing another additional axis for parallelisation. Our CUDA implementation allows for the number of tiles and tile dimensions to be adaptive at run time, where specific GPU hardware characteristics dictate the maximum image size and maximum number of tiles which can be compressed by a single kernel invocation. Parallelised tiled imagery compression can be implemented in CUDA at several different levels of the CUDA programming hierarchy, kernel or block level, as shown in Figure 5.

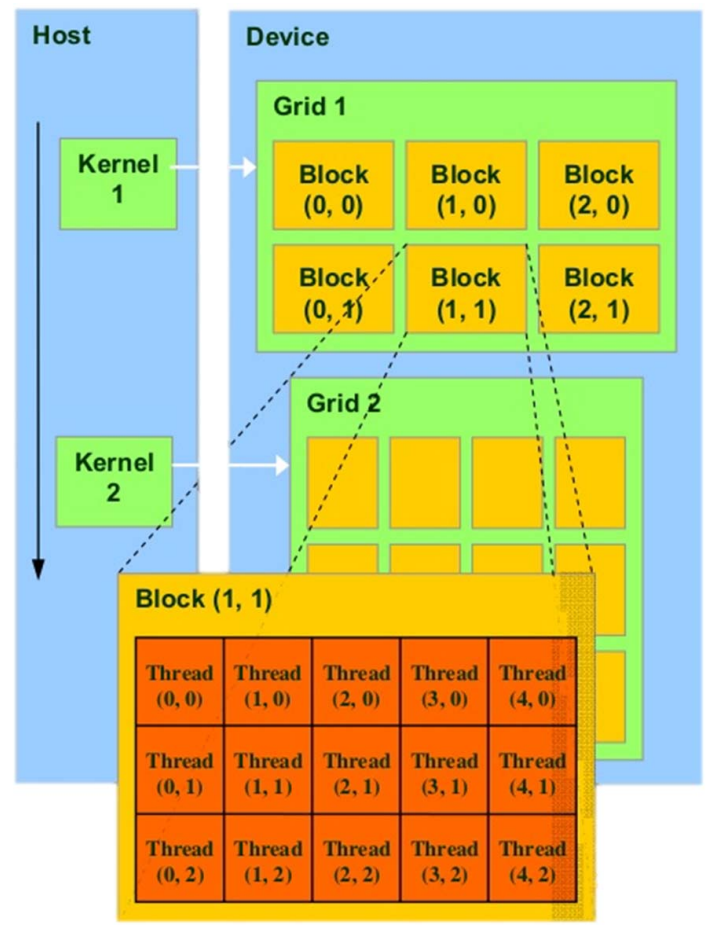

Figure 5: NVIDIA CUDA programming hierarchy [8]

CUDA 7.0 (March 2015) introduced a new concurrent programming functionality called CUDA streams [9]. This concept allows the execution of asynchronous GPU commands including host-GPU memory operations and kernel launches. Previous to CUDA 7.0 all GPU commands were allocated to the default stream and were executed synchronously with host. With the introduction of custom streams, it is now possible to execute multiple memory commands and kernel launches concurrently from the host, as GPU resources allow. This concept allows us to create an implementation in which multiple streams can be utilised to launch multiple CCSDS-123 kernels concurrently for different image tiles. The alternative approach is to implement the independent compression of multiple image tiles within a single kernel where each block of threads is tasked with the compression of each image tile. It was found 
that the compression of tiled imagery within a single kernel leveraging the highly parallel block of threads was more efficient. This is likely due to the reduced overhead of kernel launch and multiple host-GPU memory transfers as this is only required once in the later approach.

\section{Memory}

The CUDA programming model allows programmers access to several different types of memory, as shown in Figure 6 . The input image pixel sample values and output codeword and length values are stored in the global device memory. Global device memory has the largest latency of all available memory types therefore; to ensure efficient memory transactions it is important to ensure all memory operations are coalesced. Shared memory is local to each block of threads so facilitates thread cooperation and data sharing. In our CCSDS-123 kernel we utilise the on-chip low latency shared memory for the storage of the local difference and weight vector values. Registers are the fastest memory type available on the GPU, they are local to each thread and have the same lifetime as the thread. All intermediate calculation values are stored in the processor registers. The number of registers used per thread used by the kernel is 72 when compiled for SM 5.0 GPU architecture. When profiling analysis is performed on our implementation it was found that this relatively high number of registers used is the limiting factor for the performance of the kernel. As the number of register per GPU are fixed, the number of registers used limit the number of threads and the number of blocks that can be run in parallel.

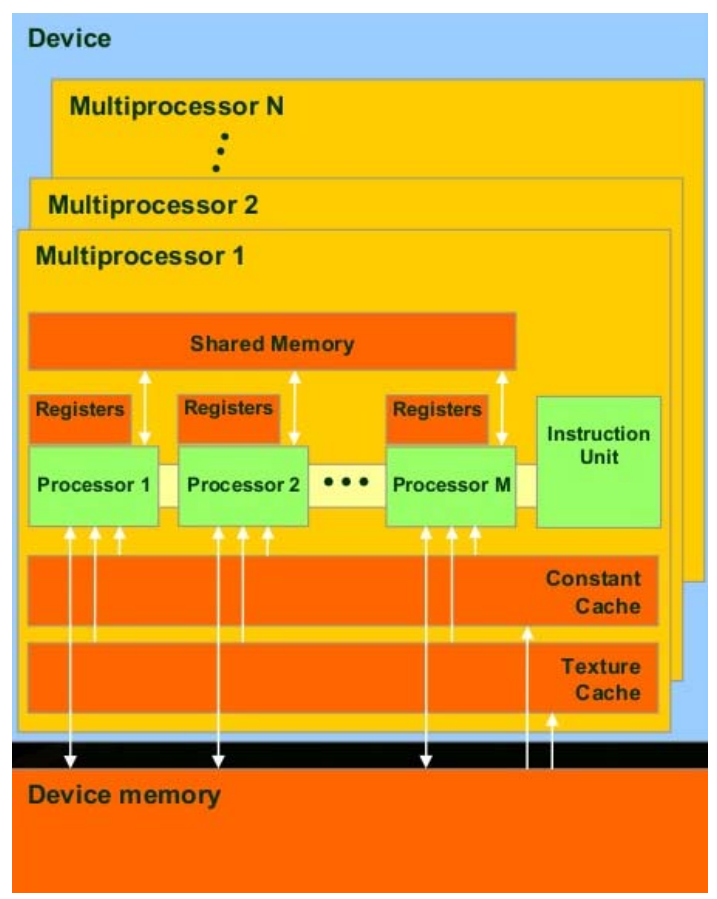

Figure 6: NVIDIA GPU memory hierarchy [8]

\section{THRUST Inclusive Sum}

THRUST is an open-source parallel algorithms library and provides a flexible high level interface for GPU optimised routines [10]. It features an abundant collection of data parallel primitives such as scan, sort, and reduce, which are key building blocks of many complex algorithms. The library is included with the CUDA toolkit and extensive documentation is available online. The THRUST algorithm can be called from both host and device code and can additionally be executed in either location where different parallelisation policies are provided. In this implementation, the THRUST Inclusive Sum algorithm was utilised to calculate the offset locations in the bitstream for the variable codewords generated from the CCSDS-123 algorithm. By calculating this offset as an inclusive sum of the individual codeword lengths, we are then able to perform the packing of the codewords into the final bitstream with no serial dependencies.

\section{Bit Packer Kernel}

The bit packer has been designed to take in the two output arrays from the CCSDS-123 kernel. The first will contain the binary variable length codeword data, represented in integer form, to be packed into an output bit stream. The second will contain the cumulatively summed, by the THRUST inclusive sum kernel, lengths of the codewords to be packed. The bit packer operation can be performed across the full number of pixels in the image as there will be no data dependencies. Therefore, each thread will be responsible for the packing of a single pixel's compressed codeword into the final bitstream. As each thread, will be working independently, it is important to ensure no two threads try and write to the same memory location at the same time therefore the atomic OR operation is used. The compressed sizes for each individual tile will also need to be stored with the bitstream to enable the decoded to identify the start and end of each tile to enable subsequent decompression.

It was found, through experimentation, that the kernel configuration of the grid of block and blocks of threads is best configured to be dependent on the GPU hardware to maximise GPU resources rather than dependent on the input image dimensions. As there are no data dependencies in the function, all data can be treated independently for maximum throughput. The compression algorithm stipulates that the output bitstream will be guaranteed to be no larger than the original image size therefore we can re-use the memory allocated to store the input pixel values for the storage of the output bitstream which eliminates an additional large memory allocation operation.

\section{Performance}

The details of the testing platform used for all original tests discussed in the paper can be found in Appendix B. The testing performed in this paper uses two images readily available online via the CCSDS image corpus [11]. The first image is the Hawaii image captured from the AVIRIS 
hyperspectral instrument and the second is the Agriculture image captured from the Landsat imager, further details on the test image attributes and the default CCSDS-123 algorithm testing parameters used can be found in Appendix C. Testing is performed for both non-tiled and tiled version of each of these images. Unless otherwise stated, the tile dimensions used for testing purposes are shown in Table 2. The performance results for the previous implementations, $[6,7]$, also used the AVIRIS Hawaii hyperspectral image. Unfortunately, not all the necessary information to facilitate a definitive comparison was provided in previous NASA publications. In addition, different GPU hardware architectures have been used, therefore a definitive comparison cannot be made between this work and previously published GPU implementations.

\section{Table 2. Tiling dimensions}

\begin{tabular}{|c|c|c|c|}
\hline Image & $\begin{array}{c}\text { Original } \\
\text { Dimensions }\end{array}$ & $\begin{array}{c}\text { Tile } \\
\text { Dimensions }\end{array}$ & $\begin{array}{c}\# \\
\text { Tiles }\end{array}$ \\
\hline AVIRIS Hawaii & $224 * 614 * 512$ & $224 * 614 * 32$ & 16 \\
\hline Landsat Agriculture & $6 * 1024 * 1024$ & $6 * 32 * 32$ & 1024 \\
\hline
\end{tabular}

Table 3 provides a summary of performance of the discussed implementation. Where the time and throughput measurements are for the whole CCSDS-123 compression chain and include device memory allocation and freeing, host to device and device to host memory transfers and the CCSDS-123, THRUST inclusive sum and bit packer kernel execution.

Table 3. New CUDA CCSDS-123 implementation performance

\begin{tabular}{|c|c|c|c|c|}
\hline Image & $\begin{array}{c}\text { Compression } \\
\text { Ratio }\end{array}$ & $\begin{array}{c}\text { Time } \\
(\mathbf{m s})\end{array}$ & $\begin{array}{c}\text { Throughput } \\
\mathbf{( M b} / \mathbf{s})\end{array}$ & Speedup \\
\hline $\begin{array}{c}\text { AVIRIS } \\
\text { Hawaii }\end{array}$ & 4.08 & 972.50 & 868.92 & $=$ \\
\hline $\begin{array}{c}\text { AVIRIS } \\
\text { Hawaii } \\
\text { Tiled }\end{array}$ & 4.03 & 175.39 & 4817.87 & 5.5 \\
\hline $\begin{array}{c}\text { Landsat } \\
\text { Agriculture }\end{array}$ & 2.01 & 2233.93 & 22.53 & $=$ \\
\hline $\begin{array}{c}\text { Landsat } \\
\text { Agriculture } \\
\text { Tiled }\end{array}$ & 2.02 & 39.60 & 1271.03 & 56.4 \\
\hline
\end{tabular}

Although testing has not currently been conducted on an extensive range of test images the initial results, shown in Table 3, confirm the expected inherent reduced compressibility and achievable processing throughput for the multispectral test image when compared to the hyperspectral test image. The results also show the significant processing throughput increase that can be achieved from performing image tiling. For the hyperspectral test image there is a speed up of approximately 5.5 times, from non-tiled to tiled tests and for the multispectral image there is an approximate speed up of 56.4 times. Despite the speed up in multispectral compression throughput achieved from performing tiling it is still approximately 3.8 times slower than the throughput achieved for the hyperspectral imagery. Therefore, the following section explores some of optimisation approaches specifically to facilitate the increased compression throughput for multispectral images.

\section{New Multispectral Optimised CUDA CCSDS-123 IMPLEMENTATION}

\section{Profiling}

To establish the current bottleneck of our CCSDS-123 CUDA implementation profiling analysis on the GPU implementation was carried out. The main bottleneck of the implementation was identified as an excessive use of registers. We have been currently unable to reduce register usage through manual code manipulation and optimisation. The NVCC CUDA compiler includes an option, called maxrregcount, to force the compiler to utilise a maximum number of registers. Various optimisations using this compile time parameter was attempted, but no increase in compression throughput was achieved.

For multispectral images, the restriction in inherent parallelism is related to the number of image bands, which for our GPU hardware implementation translates to the number of threads per block. The GPU hardware architecture is based around a SIMD or SIMT execution model whereby for current NVIDIA GPUS instructions are issued in groups of 32 threads, also known as a warp. For the Landsat agriculture test case, the image has 6 bands, this means the kernel will be launched with 6 threads per block. As instructions on the GPU are always issued in groups of 32 , whilst 32 threads will be launched only 6 will be active in the kernel. Therefore, an optimisation would to try to increase the number of threads issued per block. Due to the introduction of image tiling there are now two axes of parallelism which are consistent along the whole CCSDS-123 algorithm, bands and tiles. Therefore, we can increase the number of threads issued per block by executed the compression of multiple tiles with a single thread block. The necessary modification to our CUDA implementation was made to allow multiple tiles to be compressed with a single block of threads.

To demonstrate the impact of increasing the number of active threads per block, by compressing multiple image tiles within a single block of threads, profiling and throughput information for the CCSDS-123 kernel has been gathered and used for performance comparison. The warp execution efficiency information has been gathered from profiling testing and processing throughput is quoted for the independent CCSDS-123 kernel. This throughput measures differs from those quoted in Table 3 as it is only for the kernel execution and does not include global device memory allocations, host and GPU memory transfers or that of the 
other THRUST inclusive sum or bit packer kernel. A summary of these results can be found in Table 4 .

Table 4. New CCSDS-123 kernel efficiency

\begin{tabular}{|c|c|c|c|c|}
\hline Image & $\begin{array}{c}\text { Tiles / } \\
\text { Block }\end{array}$ & $\begin{array}{c}\text { Threads / } \\
\text { Block }\end{array}$ & $\begin{array}{c}\text { Warp } \\
\text { execution } \\
\text { efficiency }\end{array}$ & $\begin{array}{c}\text { TP } \\
\text { (Mb/s) }\end{array}$ \\
\hline $\begin{array}{c}\text { AVIRIS } \\
\text { Hawaii Tiled }\end{array}$ & 1 & 224 & $99.9 \%$ & 6010.95 \\
\hline \multirow{2}{\text{Landsat}}{$\begin{array}{c}\text { Agriculture } \\
\text { Tiled }\end{array}$} & 2 & 12 & $37.1 \%$ & 2368.26 \\
\cline { 2 - 5 } & 4 & 24 & $74.1 \%$ & 3728.27 \\
\cline { 2 - 5 } & 1 & 6 & $18.5 \%$ & 1378.90 \\
\hline
\end{tabular}

Nested Parallelism

In CUDA 5.0 (October 2012) the dynamic parallelism was introduced to the CUDA programming model [12]. This allows nested parallelism, present in an algorithm, to be exploited as kernels are now able to launch new kernels and spawn new threads on the GPU, independently from the host. Whilst previously, programmers were restricted to a flat programming model, now nested parallelism can be exploited to allow adaptive parallel implementation at run-time.

There is a small degree of nested parallelism available in our implementation of the CCSDS-123 algorithm. Therefore, we investigated the potential advantages of applying the new dynamic parallelism CUDA programming model. The nested parallelism we tried to exploit was present in the calculation of the local difference vector, the update of the weight vector and the dot product calculation of these two vectors, the impact of these functions on the number of instructions is shown in Table 5. However, in our existing implementation the weight and local difference vectors are stored in the low latency on-chip shared memory. This memory is local to a single kernel block of threads and as launching a new kernel creates a new thread block, shared memory cannot be used to pass data between nested kernels. The final dynamically parallelised version of the CUDA CCSSD-123 implementation achieved a significantly lower throughput than the previous version. We postulate that this is because the amount of nested parallelism is too small to overcome the both the additional kernel launch overhead and the performance loss from not being able to utilise the low latency shared memory.

\section{Performance}

Our final multispectral optimised CUDA CCSDS-123 implementation only varies from our original CUDA implementation by the introduced ability to be able to compress multiple tiles within a single thread block. The results for this multispectral optimised solution are shown in Table 6. These results show that whilst compressing multiple tiles with a thread block is slightly detrimental to the processing throughput for the hyperspectral image, we can achieve up to a 2.41 speed up for the multispectral image.

Table 5. Instruction count of weight \& local difference vector operations

\begin{tabular}{|c|c|c|}
\hline Function & $\begin{array}{c}\text { Instruction } \\
\text { Execution } \\
\text { Count }\end{array}$ & $\begin{array}{c}\text { \% Max } \\
\text { Execution } \\
\text { Count }\end{array}$ \\
\hline $\begin{array}{c}\text { Dot Product of Weight } \\
\text { \& Local Difference } \\
\text { Vectors }\end{array}$ & 125426448 & 100 \\
\hline Weight Vector Update & 125426448 & 100 \\
\hline $\begin{array}{c}\text { Local Difference } \\
\text { Vector Calculations }\end{array}$ & 52811136 & 42 \\
\hline
\end{tabular}

Table 6. New multispectral optimised CCSDS-123 CUDA implementation performance

\begin{tabular}{|c|c|c|c|c|c|}
\hline Image & $\begin{array}{c}\text { Tiles / } \\
\text { Block }\end{array}$ & $\begin{array}{c}\text { Threads } \\
\text { / Block }\end{array}$ & $\begin{array}{c}\text { Time } \\
(\mathbf{m s})\end{array}$ & $\begin{array}{c}\text { Throughput } \\
\text { (Mb/s) }\end{array}$ & $\begin{array}{c}\text { Speed } \\
\text { up }\end{array}$ \\
\hline \multirow{2}{*}{$\begin{array}{c}\text { AVIRIS } \\
\text { Hawaii } \\
\text { Tiled }\end{array}$} & 1 & 224 & 175.39 & 4817.87 & $=$ \\
\cline { 2 - 6 } & 1 & 448 & 178.65 & 4730.15 & -1.02 \\
\hline \multirow{2}{*}{$\begin{array}{c}\text { Landsat } \\
\text { Agriculture } \\
\text { Tiled }\end{array}$} & 4 & 24 & 16.44 & 3061.18 & 2.41 \\
\cline { 2 - 6 } & 8 & 48 & 16.76 & 3002.26 & 2.36 \\
\cline { 2 - 6 } & 16 & 96 & 17.15 & 2934.88 & 2.31 \\
\hline
\end{tabular}

\section{EXPERIMENTAL STUDIES}

\section{Input Parameter Experiments}

There are 12 tuneable user imputable parameters to the standard CCSDS-123 algorithm, three of these have been shown to have the greatest impact on execution time and compression ability in [7]. These are the number of prediction band and the prediction and local sum modes. The number of prediction bands used by the prediction algorithm is a user definable parameter which can be adjusted at runtime. It determines the number of preceding spectral bands which used in the local neighbourhood calculations in the prediction stage of the CCSDS-123 algorithm. 
The different prediction and local sum modes allow the compression algorithm to be easily adapted to suit different image type. It is common that many raw uncalibrated pushbroom imager data often exhibit streaking artefacts the reduced prediction mode and column-orientated local sum mode allow the algorithm to only utilise pixel from the same along-track position in the image increasing the compression ratio performance. In addition, these mode's feature less computation therefore likely achieved increased processing throughput. For calibrated or images that do not exhibit streaking artefacts the full prediction mode and neighbourorientated local sum mode will often provide an increase in achieved compression ratio.

The results from our study into the impact of changing number of prediction bands and the influence of the prediction and local sum modes on compression ratio and processing throughput are given for both AVIRIS Hawaii and Landsat Agriculture test images in Figure 7 and Figure 8 respectively. In these tests, the term reduced mode is used to refer to the use of both the reduced prediction mode and column-orientated local sum mode. Whilst full mode refers to the use of both full prediction mode and neighbourorientated local sum mode.

It is clear from the results of both test images the impact the number of prediction band has on both the compression ratio and throughput. As the number of prediction bands has a positive influence on the number of executed instructions, as the number of prediction bands increases the processing throughput decreases. However, increasing the number of preceding band also increases the amount of spectral redundancy available for exploitation, resulting in an increase in compression ratio. Therefore, there is a trade-off between throughput and compression ratio which needs to be made in advance. For both images selecting between $1-3$ prediction bands provide the best trade-off between maximising both compression ratio and throughput.

For the study of the impact of the prediction and local sum modes the results show that for both test image the full modes provide the best compression ratio performance, this would suggest that both test images do not feature any streaking artefacts that require these specialised modes. With regards to the mode choices impact on the processing throughput for between 1-3 prediction band we could expect the throughput reduction from reduced to full modes to be the approximately $500 \mathrm{Mb} / \mathrm{s}$ for the AVIRIS image and approximately $200 \mathrm{Mb} / \mathrm{s}$ for the Landsat image. Testing should be completed with a more extensive set of test imagery to gain a full picture of the trends and influences of these parameters on both compression ratio and throughput performance.

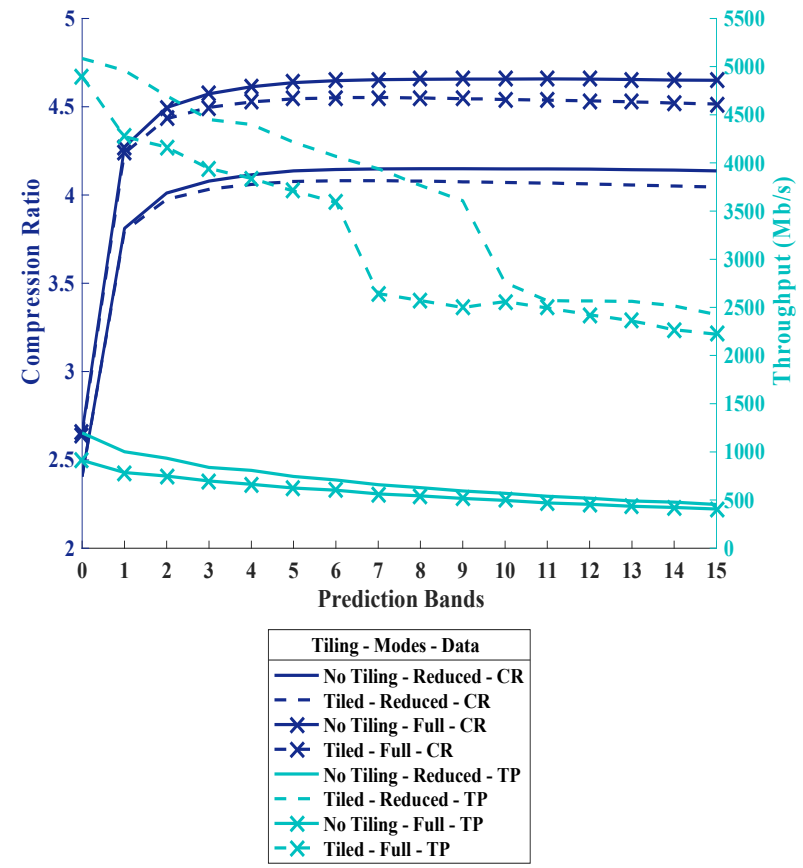

Figure 7: AVIRIS Hawaii input parameter testing results

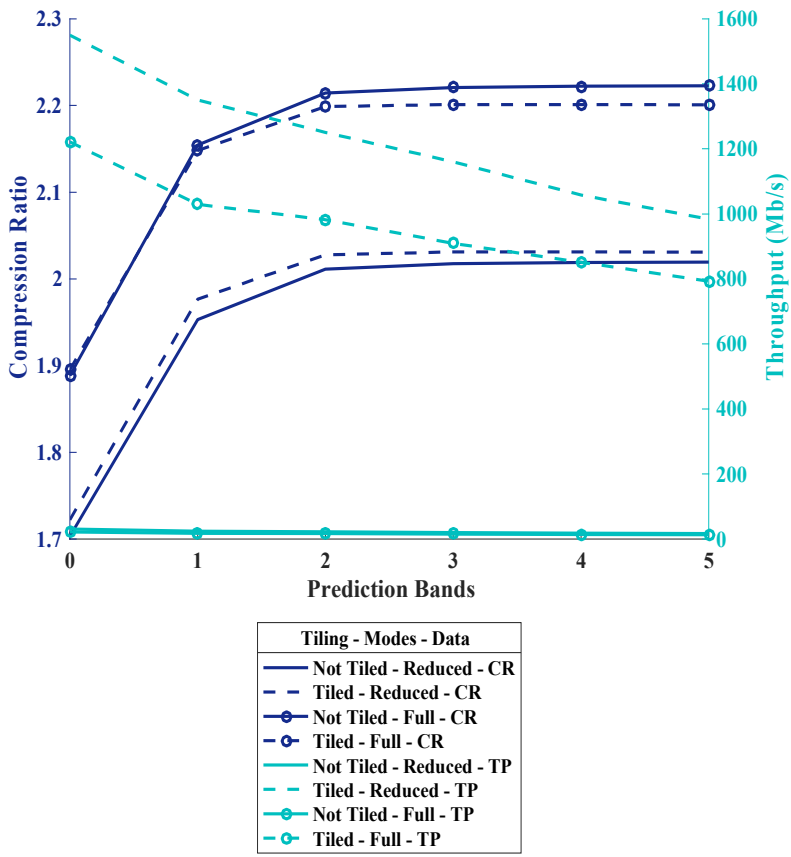

Figure 8: Landsat Agriculture input parameter testing results

\section{Tile Dimensionality Testing}

A study into the impact of the choice of size and dimensions for image tiling has also been conducted. This study will help to establish trends in the relationship between 
tile dimensions and compression ratio and throughput. Understanding these relationships is key to ensure that the selection of tile size for image compression helps to achieve a suitable trade-off between compression ratio and throughput. Due to the nature of the original image AVIRIS Hawaii dimensions the test image has been tiled only in the $\mathrm{Y}$ dimension to gives tiles as a number of image lines, these results are given in Figure 9. Whilst the Landsat test image has been tiled into square blocks and these results are given in Figure 10.

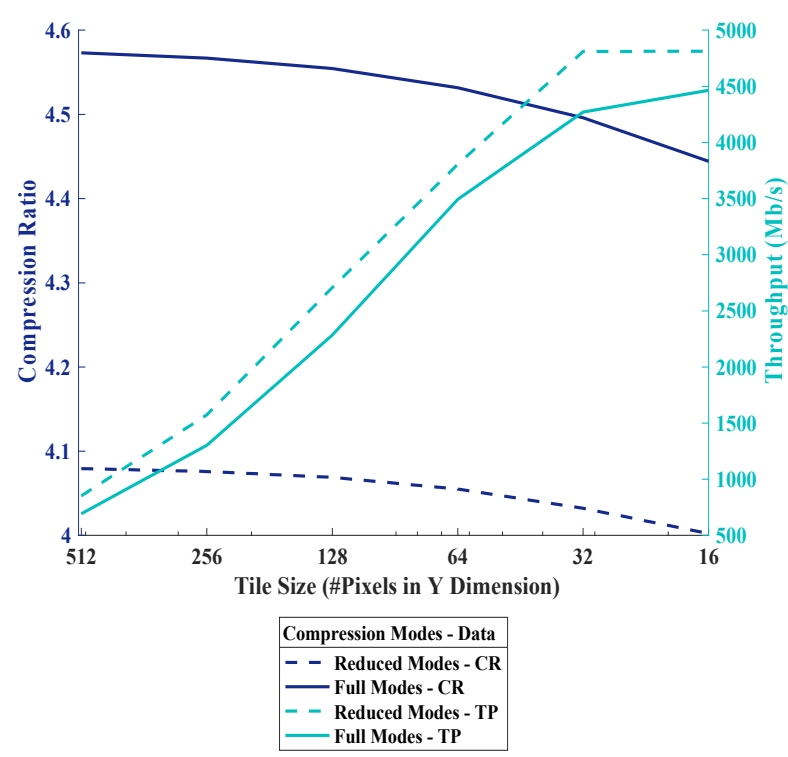

Figure 9: AVIRIS Hawaii tile size testing

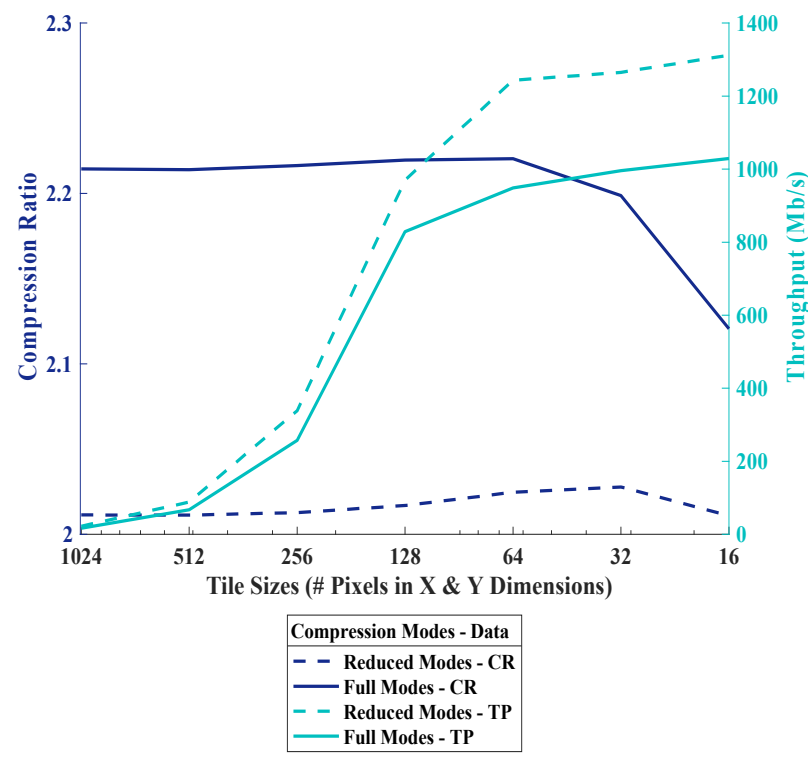

Figure 10: Landsat Agriculture tile size testing
Both the tiling studies conducted show similar trends between the decrease in tile size and subsequent increase in compression throughput, where the margin of throughput gain reduces as tile size gets smaller. What differs between the two test images is the slight difference in impact on reducing tile size on the compression ratio, this gives more unique selection of tile size to provide the best trade-off between compression ratio and throughput. For the tile size selection for both maximised compression and throughput for the AVIRIS image this would be a size of 32 in the Y dimension regardless of compression mode. Whilst for the Landsat image this would a size of 128 for the reduced compression modes and either 64 or 32 for full compression modes.

The final experimentally results presented in this paper show the impact of the tuneable tiles per block parameter of the new multispectral imagery optimised CUDA CCSDS-123 implementation, and are given in Figure 11. This plot shows that for the multispectral Landsat Agriculture test image implementing the compression of at least 2 tiles per block always achieves greater compression than the case of the unoptimised case where only 1 tile is compressed per block. Although the overall best number of tiles per block number for greatest throughput is dependent on tile size.

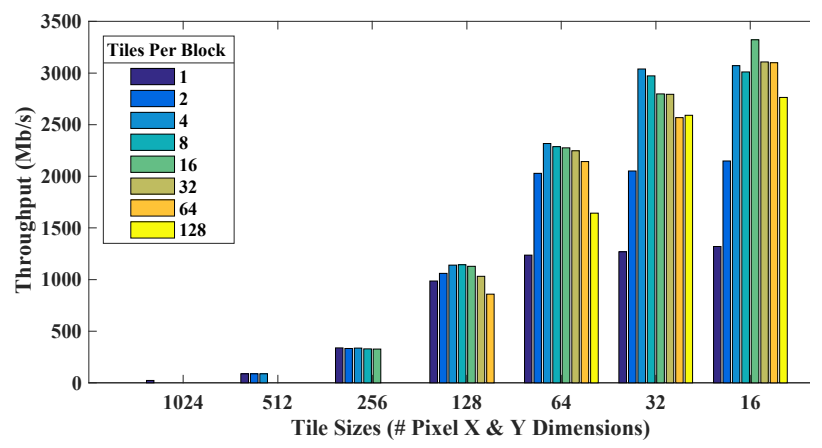

Figure 11: Landsat Agriculture tile size and multispectral optimisation parameter testing

\section{FUTURE WORK}

Onboard Hardware Architecture Demonstration

Work is underway to develop a test setup which is representative of the proposed new heterogeneous onboard hardware architecture as shown in Figure 3. This will then be used to demonstrate and perform performance analysis of the CUDA GPU implementation of the CCSDS-123 algorithm discussed in this work and the FPGA streaming data interfaces, buffering and data formatting tasks.

\section{Radiation Hardening By Software Design}

Currently, GPU's are not manufactured to provide radiation tolerance at the silicon gate level. This poses an obstacle in the practical deployment of a GPU device in an onboard system as it conflicts with the traditional ethos 
within the space industry, to strictly utilise only radiation hardened processors. Therefore, Radiation Hardening by Software Design (RHBSW) principles will be adapted and implemented in the proposed system to ensure radiation effects are mitigated $[13,14,15]$. Some RHBSW techniques which have been proposed in literature to date include: heartbeat monitoring, watchdog timers, control flow assertions, progress monitoring and checkpoint and rollback functionality. The highly parallel nature of the GPU could also allow for the adaption of traditional space modular redundancy techniques, such as triple modular redundancy (TMR), for GPU software.

\section{SUMMARY}

In this paper two new CUDA GPU implementations of the multidimensional lossless image compression algorithm CCSDS-123 has been presented. The first implementation leverages the low-latency on-chip shared memory for the manual caching of several key intermediate compression variables. This and the exploitation of additional parallelism introduced by image tiling facilitates the high processing throughput achieved for both hyperspectral and multispectral test images. The results given in Table 3 gives an overview of the performance of our first implementation. The confirm our initial thoughts that both compression ratio and throughput would be reduced for the multispectral test image when compared to the hyperspectral test image due to underlying image characteristics. The results also highlight the significant throughput increase that can be achieved from performing image tiling. For the hyperspectral image a speedup of approximately 5.5 times was achieved for the tiled image whilst for the multispectral image there was a considerable speedup of approximately 56.4 times.

However, despite the considerable speedup achieved from the tiling of the multispectral image, the compression throughput achieved is still approximately 3.8 times slower than the throughput achieved for the hyperspectral imagery. This provided the motivation to explore the optimisation of the implementation specifically for multispectral data sets. The second implementation increases the utilisation of the underlying GPU hardware architecture for multispectral EO imagery, as shown in Table 4. As a result, we are able to achieve a peak processing throughput of $2934.88 \mathrm{Mb} / \mathrm{s}$ a 2.3 times speedup on the first implementation for the same image and tiling parameters. This makes the multispectral optimised CUDA implementation now only approximately 1.6 times slower than the throughput achieved for the hyperspectral test image.

\section{APPENDICES}

\section{A. CCSDS-123 IMPLEMENTATION FEATURES}

\begin{tabular}{|l|c|c|}
\hline Description & Status & Supported? \\
\hline Signed Samples & $\mathrm{O} .1$ & $\mathrm{~N}$ \\
\hline Unsigned Samples & $\mathrm{O} .1$ & $\mathrm{Y}$ \\
\hline X Size, $N \mathrm{X}$ & $\mathrm{M}$ & $\mathrm{Y}$ \\
\hline
\end{tabular}

\begin{tabular}{|c|c|c|}
\hline Y Size, $N Y$ & $\mathrm{M}$ & $\mathrm{Y}$ \\
\hline Z Size, $N Z$ & $\mathrm{M}$ & $\mathrm{Y}$ \\
\hline Dynamic Range, $D$ & $\mathrm{M}$ & $\mathrm{Y}$ \\
\hline $\begin{array}{l}\text { Number of Prediction Bands, } \\
P\end{array}$ & M & $\mathrm{Y}$ \\
\hline Full Prediction Mode & 0.2 & $\mathrm{Y}$ \\
\hline Reduced Prediction Mode & 0.2 & $\mathrm{Y}$ \\
\hline $\begin{array}{l}\text { Neighbour-Oriented Local } \\
\text { Sums }\end{array}$ & O.3 & Y \\
\hline Column-Oriented Local Sums & 0.3 & $\mathrm{Y}$ \\
\hline $\begin{array}{ll}\text { Weight } & \text { Component } \\
\text { Resolution, } \Omega & \\
\end{array}$ & M & Y \\
\hline Default Weight Initialization & 0.4 & $\mathrm{Y}$ \\
\hline Custom Weight Initialization & 0.4 & $\mathrm{~N}$ \\
\hline $\begin{array}{ll}\text { Weight } & \text { Initialization } \\
\text { Resolution, } Q & \\
\end{array}$ & C. 21 & $\mathrm{~N}$ \\
\hline Register Size, $R$ & $\mathrm{M}$ & $\mathrm{Y}$ \\
\hline $\begin{array}{llr}\text { Weight } & \text { Update } & \text { Scaling } \\
\text { Exponent } & \text { Initial Parameter, } \\
\text { vmin } & \\
\end{array}$ & M & $\mathrm{Y}$ \\
\hline $\begin{array}{llr}\text { Weight } & \text { Update } & \text { Scaling } \\
\text { Exponent } & \text { Final Parameter, } \\
\text { vmax } & & \\
\end{array}$ & M & $\mathrm{Y}$ \\
\hline $\begin{array}{llr}\text { Weight } & \text { Update } & \text { Scaling } \\
\text { Exponent } & \text { Change } & \text { Interval, } \\
\text { tinc } & & \\
\end{array}$ & M & $\mathrm{Y}$ \\
\hline Compressed Image & $\mathrm{M}$ & $\mathrm{Y}$ \\
\hline Output Word Size, $B$ & $\mathrm{M}$ & $\mathrm{Y}$ \\
\hline Header & $\mathrm{M}$ & $\mathrm{Y}$ \\
\hline $\begin{array}{l}\text { Weight Initialization Table } \\
\text { Encoding }\end{array}$ & C. 31 & $\mathrm{~N}$ \\
\hline Compressed Image Body & $\mathrm{M}$ & $\mathrm{Y}$ \\
\hline BI Encoding Order & 0.5 & $\mathrm{Y}$ \\
\hline BSQ Encoding Order & O.5 & $\mathrm{N}$ \\
\hline $\begin{array}{ll}\text { Sub-frame } & \text { Interleaving } \\
\text { Depth, } M & \\
\end{array}$ & C. 32 & $\mathrm{Y}$ \\
\hline $\begin{array}{ll}\text { Sample-Adaptive } & \text { Entropy } \\
\text { Coder } & \\
\end{array}$ & 0.6 & $\mathrm{Y}$ \\
\hline $\begin{array}{l}\text { Block-Adaptive } \\
\text { Coder }\end{array}$ & O.6 & $\mathrm{N}$ \\
\hline $\begin{array}{l}\text { Accumulator Initialization } \\
\text { Table Encoding }\end{array}$ & $\mathrm{O}$ & $\mathrm{N}$ \\
\hline Initial Count Exponent, $\gamma 0$ & $\mathrm{M}$ & $\mathrm{Y}$ \\
\hline $\begin{array}{ll}\text { Accumulator } & \text { Initialization } \\
\text { Constant, } K & \\
\end{array}$ & $\mathrm{O}$ & Y \\
\hline $\begin{array}{l}\text { Rescaling Counter Size, } \\
\gamma^{*}\end{array}$ & M & $\mathrm{Y}$ \\
\hline Unary Length Limit, $U \max$ & $\mathrm{M}$ & $\mathrm{Y}$ \\
\hline
\end{tabular}

O.1: It is mandatory to support at least one of these items. O.2: When $N \mathrm{X}=1$, support is mandatory for Reduced Prediction Mode and not applicable for Full Prediction Mode. Otherwise, it is mandatory to support at least one of these items.

O.3: When $N \mathrm{X}=1$, support is mandatory for ColumnOriented Local Sums and not applicable for NeighbourOriented Local Sums. Otherwise, it is mandatory to support 
at least one of these items.

O.4: It is mandatory to support at least one of these items. C.21: If Custom Weight Initialization supported - then mandatory

C.31: If Custom Weight Initialization supported - then optional

C.32: If BI Encoding Order supported - then mandatory

O.6: It is mandatory to support at least one of these items.

\section{B. TeSt Platform SPECIFICATION}

\begin{tabular}{|l|l|}
\hline Desktop & Dell OPTIPLEX 7010 (SMF) \\
\hline CPU \& Clock & Intel Core i5-3470 @ 3.20GHz \\
\hline CPU RAM & 4GB DDR3 \\
\hline GPU \& Clock & $\begin{array}{l}\text { NVIDIA GeForce GTX 750Ti } \\
@ 33 \mathrm{MHz}\end{array}$ \\
\hline GPU RAM & 2GB GDDR5 \\
\hline
\end{tabular}

\section{Test Images \& Default Testing PARAMETERS}

\begin{tabular}{|c|c|c|}
\hline Image Name & AVIRIS Hawaii & Landsat Agriculture \\
\hline Height & 512 & 1024 \\
\hline Width & 614 & 1024 \\
\hline Bands & 224 & 6 \\
\hline Bit Depth & 12 & 8 \\
\hline $\begin{array}{l}\text { File Size } \\
\text { (Bytes) }\end{array}$ & $105,627,648$ & $6,291,456$ \\
\hline $\begin{array}{l}\text { Prediction } \\
\text { Bands }\end{array}$ & 3 & 2 \\
\hline $\begin{array}{l}\text { Prediction } \\
\text { Mode }\end{array}$ & Reduced & Reduced \\
\hline $\begin{array}{l}\text { Local Sum } \\
\text { Mode }\end{array}$ & $\begin{array}{l}\text { Column- } \\
\text { Orientated }\end{array}$ & Column-Orientated \\
\hline $\begin{array}{l}\text { Register } \\
\text { Size }\end{array}$ & 64 & 64 \\
\hline $\begin{array}{l}\text { Weight } \\
\text { Resolution }\end{array}$ & 19 & 19 \\
\hline $\begin{array}{l}\text { Weight } \\
\text { Interval }\end{array}$ & 6 & 6 \\
\hline $\begin{array}{l}\text { Weight } \\
\text { Initial }\end{array}$ & -1 & -1 \\
\hline $\begin{array}{l}\text { Weight } \\
\text { Final }\end{array}$ & 3 & 3 \\
\hline U Max & 18 & 18 \\
\hline Y Star & 6 & 6 \\
\hline Y Zero & 2 & 2 \\
\hline $\mathrm{K}$ & 2 & 2 \\
\hline
\end{tabular}

D. Keymeulen , N. Aranki , B. Hopson , A. Kiely , M Klimesh and K. Benkrid, "GPU lossless hyperspectral data compression system for space applications", Proc. 2012 IEEE Aerospace Conf., pp. 1-9, 2012

[7] B. Hopson, K. Benkrid, D. Keymeulen, N. Aranki, "Realtime CCSDS lossless adaptive hyperspectral image compression on parallel GPGPU \& multicore processor systems", Proc. 2012 NASA/ESA Conf. Adaptive Hardware and Systems (AHS), pp. 107-114, 2012-Jun.

[8] C. Zeller, "Tutorial CUDA", NVIDIA Developer Technology, NVIDIA Corporation, April 2008, (Accessed October 2016)

[9] M. Harris, "GPU Pro Tip: CUDA 7 Streams Simplify Concurrency",

https://devblogs.nvidia.com/parallelforall/gpu-pro-tip-cuda7-streams-simplify-concurrency/, January $22 \quad 2015$, (Accessed October 2016)

[10] NVIDIA CUDA Toolkit Documentation V7.0. http://docs.nvidia.com/cuda/index.html. (Accessed October 2016)

[11] CCSDS MHDC - Space Link Services Image Compression Testing Corpus http://cwe.ccsds.org/sls/docs/Forms/AllItems.aspx?RootFol der $=\% 2 \mathrm{Fsls} \% 2 \mathrm{Fdocs} \% 2 \mathrm{FSLS} \% 2 \mathrm{DDC} \% 2 \mathrm{~F} 123 \% 2 \mathrm{E} 0 \% 2 \mathrm{DB}$ $\% 2 \mathrm{DInfo} \% 2 \mathrm{FTestData} \& \mathrm{View}=\%$ 7B16ACDA38\%2DFFA3 $\% 2 \mathrm{D} 4657 \% 2 \mathrm{D} 8 \mathrm{~F} 27 \% 2 \mathrm{DB} 166 \mathrm{C} 23 \mathrm{C} 24 \mathrm{~A} 2 \% 7 \mathrm{D} \& \quad, \quad J u n e$ 2016 (Accessed October 2016)

[12] A. Adinetz, "Adaptive Parallel Computation with CUDA Dynamic Parallelism", 
https://devblogs.nvidia.com/parallelforall/introduction-cudadynamic-parallelism/, May 6 2014, (Accessed October 2016)

[13] A. G. Schmidt, J. P. Walters, K. M. Zick, M. French, D. Keymeulen, N. Aranki, M. Klimesh, and A. Kiely, "Applying radiation hardening by software to fast lossless compression prediction on FPGAs," in Proceedings of the 2012 IEEE Aerospace Conference, March 2012.

[14] A. Schmidt and M. French, "Fast lossless image compression with radiation hardening by hardware/software co-design on platform FPGAs", Application-Specific Systems, Architectures and Processors (ASAP), 2013 IEEE 24th International Conference on, pp. 103-106

[15] H. Takizawa, K. Sato, K. Komatsu, and H. Kobayashi, "CheCUDA: A Checkpoint/Restart Tool for CUDA Applications," In Proceedings of International Conference on Parallel and Distributed Computing, Applications, and Technologies (PDCAT), pp. 408-413, 2009.

\section{BIOGRAPHY}

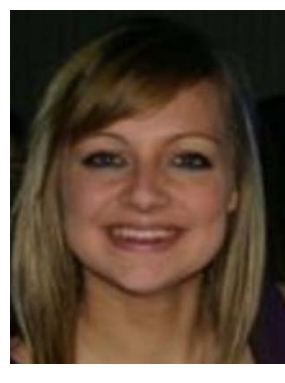

Rebecca L. Davidson received a BEng in Electronic Engineering from the University of Surrey, in 2014. She is currently a PhD student at Surrey Space Centre, University of Surrey. Her current research is in onboard data processing for downlink optimisation of multispectral Earth observation satellites. Her current focus is upon efficient hardwaresoftware co-design utilising commercial-off-the-shelf processors for space.

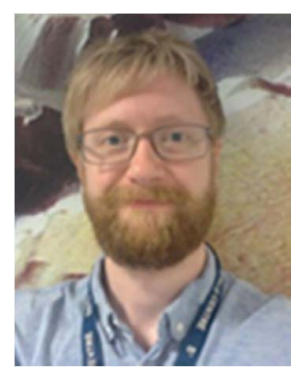

Dr Christopher P. Bridges (BEng, 2005; PhD 2009) leads the On-Board Data Handling (OBDH) research group within Surrey Space Centre (SSC). He researches software defined radios, real-time embedded systems, agent computing, Java processing, multi-core processing in FPGAs, and astrodynamics computing methods in many spaceflight payloads. In 2013, he designed, built and still operates the UK's first CubeSat (STRaND-1) with SSTL and now contributes towards computing hardware and software in missions with SSTL, on ESA's ESEO mission and also the NASA-JPL/CalTech AAReST mission. 